\title{
Assessment of the entomopathogenic nematode bacteria against the termite, Microtermes mycophagus D. (Isoptera: Termitidae)
}

\author{
Erum Yawar Iqbal', Umair Ali Memon, Syeda Faryal Zehra Abidi, Saboohi Raza and Shahina Fayyaz
}

\begin{abstract}
The capability of symbiotic bacteria of entomopathogenic nematodes against the termite, Microtermes mycophagus D. (Isoptera: Termitidae), was assessed. Different fractions of Pakistani isolates of entomopathogenic bacteria viz., Xenorhabdus indica strain (Pak.S.B.50), X. indica strain (Pak.S.B.56), X. stockiae strain (Pak.S.B. 65), and X. steinernematis strain (C.B.10) were assessed against M. mycophagus by direct contact method (spraying method) and sand assay in laboratory conditions. Mortality response of cell-free filtrates after $24 \mathrm{~h}$ at $20^{\circ} \mathrm{C}$ for $X$. indica (Pak.S.B.50), $T_{2}=X$. indica (Pak.S.B.56), $T_{3}=X$. stockiae (Pak.S.B. 65), and $T_{4}=X$. steinernematis (C.B.10) ranged (88.3-100\%) as $33 \pm 9.34,98.33 \pm$ $6.22,88.33 \pm 7.22$, and $100.00 \% \pm 0.00$, respectively. In the case of sand assay, the most effective treatment was $T_{4}$, where (100\%) mortality rate was recorded $24 \mathrm{~h}$ post application of B.S. (bacterial suspension) $\left(4 \times 10^{4} \mathrm{CFU} / \mathrm{ml}\right)$ and CFF (cell-free filtrate) $(100 \mu \mathrm{l} / 10 \mathrm{ml})$ at 20 and $25^{\circ} \mathrm{C}$.
\end{abstract}

Keywords: Entomopathogenic bacteria, Xenorhabdus, Termite, Microtermes mycophagus, Bacterial fractions, Biocontrol

\section{Background}

The termite, Microtermes mycophagus D. (Isoptera: Termitidae: Macrotermitinae), is a cosmopolitan pest of wood and wood products that can be distinguished by its colonial behavior. Colony members are distinctly varied morphologically, i.e., propagative (king and queen), soldiers, and workers. The head termites, the king and queen, are sexually functional but pheromonal regulation that is responsible for the caste production is only produced by the queen (Noirot and Noirot-Timothee, 1970). Wingless individuals, workers or soldiers, are usually non-reproductive males or females. Soldiers play a major role to defend the colony and represent $1 / 10$ th of the population of a colony (Bignell and Eggleton, 1998). Termites are highly devastating and cause damage to furniture, buildings, trees, and

\footnotetext{
* Correspondence: erum i@yahoo.com

National Nematological Research Centre, University of Karachi, Karachi 75270,
} Pakistan

agricultural crops, such as cereals, oil crops, pulses, sugarfruits, and vegetables. Estimated losses by this pest and Termes, are the most prevalent termites in Pakistan Manzoor and Naeem, 2010). gants containing methyl bromide, sulfuric fluoride, or a combination of carbon dioxide and methyl bromide is the suitable procedure of eradicating dry wood termites. Biocontrol agents are environment friendly and proficient in working but its cost effective feature is debatable. Abiotic factors such as warm and moist favored by subterranean termites, which promote epizootics, also have the potenfor biological control (Verma et al., 2009). Few studies have reported the potential of entomopathogenic nematodes (EPN) to control termites. EPN exposure to termites 
resulted in significant response of parasitization ( $47 \%$ after 4 days) and $100 \%$ mortality after 12 days under lab conditions. Fujii (1975) gained $96 \%$ mortality results of C. formosanus within 7 days after treating with infective-stage Steinernema carpocapsae (Weiser) (Steinernematidae) in laboratory analysis. Mortality rate, more than $95 \%$, was documented within 3 days by Georgis et al., (1982) for both Reticulitermes sp. and Zootermopsis sp. after laboratory exposure to $S$. carpocapsae; further termites were also serve as vectors for EPN that take back to their colonies. S. carpocapsae has shown high rates of infection to Nasutitermes costalis and $R$. flavipes obtained under laboratory conditions (Laumond et al., 1979 and Trudeau, 1989).

The present study aimed to assess the efficacy of symbiotic bacteria Xenorhabdus species as biopesticide against the termite, $M$. mycophagus, under laboratory conditions.

\section{Materials and methods}

\section{Collection of termite}

The termite (M. mycophagus) was collected from different infested trees in the premises of the University of Karachi, Karachi (24 $56^{\prime} 21.833^{\prime \prime}$ N, 67 $7^{\prime} 14.869^{\prime \prime}$ E), Pakistan.

\section{Bacterial culture (isolation of bacteria from insect hemolymph)}

Entomopathogenic nematodes (EPN) were obtained from the storage unit, maintained by Prof. Dr. Shahina Fayyaz at NNRC, University of Karachi, Karachi, Pakistan. All nematodes were propagated in last instar larvae of the greater wax moth, Galleria mellonella L., using the method of Dutky (1974). Infective juveniles were collected by White traps (White, 1927), harvested, and stored in sterilized distilled water at $10-15^{\circ} \mathrm{C}$ for no more than 2 weeks before they were used.

\section{Isolation of bacteria from insect hemolymph}

To isolate bacteria from hemolymph, G. mellonella larvae were inoculated by EPN (Table 1) 100 IJs in a Petri dish lined with moistened filter paper. After $48 \mathrm{~h}$ the dead larvae were surface sterilized by $75 \%$ ethanol for $15 \mathrm{~min}$; then, the cadavers were passed through the flame for further sterilization. Cadavers were dissected with sterilized scissors at the second foot, a loop full of hemolymph streaked onto NBTA agar medium (Akhurst, 1980). The streaked plates were incubated in the dark at $28^{\circ} \mathrm{C}$ for $48 \mathrm{~h}$ for the development of primary colonies. For further purification, single colonies of bacteria were sub cultured on new plates of agar medium. Then, single colony was transferred to the nutrient broth ( 0.81 broth $+61 \mathrm{ml}$ water) and kept it for incubation on shaking bath for 2 days at $150 \mathrm{rpm} \mathrm{ND}$ $28^{\circ} \mathrm{C}$. The bacterial suspension was used for bioassay.

\section{Biochemical analysis of bacterial isolates}

The pure cultures of different species of Xenorhabdus were subjected to biochemical test through API 20E test kit of Biomerieux Ltd., USA.

\section{Effect of different application method for controlling the termite}

Pakistani isolates of entomopathogenic bacteria viz., Xenorhabdus indica (Pak.S.B.50), X. indica (Pak.S.B.56), X. stockiae (Pak.S.B. 65), and X. steinernematis (C.B.10) were assessed against $M$. mycophagus by direct contact method (spraying method) and sand assay in a laboratory experiment.

\section{Spray method}

Heavy infested branches of different trees were selected and kept in a plastic shopper after cutting with a hammer or cutter and brought in to the laboratory. Plastic containers about the size of $8 \times 6$ in. lined with wax at the edges were used for the experiment. Six-inch pieces of tree branches carrying approximately 50 termite individuals were placed in each container and sprayed with $20 \mathrm{ml}$ of each treatment separately. Each container was sealed with a parafilm and each set of experiment incubated at different temperatures 20,25 , and $30^{\circ} \mathrm{C}$. Different EPB formulations were examined viz., B.S. (bacterial suspension) $\left(4 \times 10^{4} \mathrm{CFU} / \mathrm{ml}\right)$, CFF (cell-free filtrate) $(100 \mu \mathrm{l} / 10 \mathrm{ml})$, B.R. (bacterial residue) $(100 \mu \mathrm{l} / 10 \mathrm{ml})$ of $T_{1}$ (Xenorhabdus indica (Pak.S.B.50)), $T_{2}$ (X. indica (Pak.S.B.56)), $T_{3}$ (X. stockiae (Pak.S.B. 65)), and $T_{4}(X$. steinernematis (C.B.10)). This combination was replicated 3 times with control treatment, which was sprayed only with water. Mortality rate was assessed each after 24,48 , and $72 \mathrm{~h}$.

\section{Sand barrier assay}

A set of 50 termite individuals was placed in $6 \times 6$ in. Petri dish lined with a filter paper. For sand assay, a

Table 1 Details of symbiotic bacteria used for the study

\begin{tabular}{lllll}
\hline Strains & Bacterial symbionts & EPN & Accession no. & Authority* \\
\hline Pak.S.B.50 & Xenorhabdus indica & Steinernema abbasi & MF498486 & Shahina F. \& Salma J. \\
Pak.S.B.56 & Xenorhabdus indica & S. pakistanense & MF521953 & Shahina F. \& Salma J. \\
Pak.S.B. 65 & Xenorhabdus stockiae & S. siamkayai & MF521964 & Shahina F. \& Salma J. \\
Pak.C.B. 10 & Xenorhabdus steinernematis & S. maqbooli & KU097324 & Shahina F. \& Salma J. \\
\hline
\end{tabular}

*Accession no. authorized by these persons 
Table 2 Biochemical analysis of symbiotic bacteria

\begin{tabular}{lllll}
\hline Biochemical analysis & Xenorhabdus indica Pak.S.B.50 & X. indica Pak.S.B.56 & X. stockiae Pak.S.B. 65 & X. steinernematis CB.10 \\
\hline Citrate utilization & + & + & - & + \\
Esculin hydrolysis & ++ & + & - & + \\
Catalase & + & ++ & - & + \\
Meso-inositol fermentation & + & - & - & - \\
Salicin fermentation & + & - & - & + \\
Ribose fermentation & + & - & - & + \\
Lipase tween 80 & + & + & & \\
\hline
\end{tabular}

"+" 90-100\% positive, "-" 90-100\%, "++" positive 75-89\%, "- -" 75-89\%, "d" positive $25-74 \%$ of strains

thin film of autoclaved sand was spread over the filter paper and then the termites were placed. Twenty milliliters of different formulations B.S. (bacterial suspension) $\left(4 \times 10^{4} \mathrm{CFU} / \mathrm{ml}\right)$, CFF (cell free filtrate) $(100 \mu \mathrm{l} /$ $10 \mathrm{ml})$, B.R. (bacterial residue) $(100 \mu \mathrm{l} / 10 \mathrm{ml})$ of $T_{1}$ (Xenorhabdus indica (Pak.S.B.50)), $T_{2}$ (X. indica (Pak.S.B.56)), $T_{3}$ (X. stockiae (Pak.S.B. 65)), and $T_{4}(X$. steinernematis (C.B.10)) along with $1 \mathrm{ml}$ of $2 \%$ Tween 80 (as emulsifier) were dropped on the sand layer under the laminar flow cabinet. Plates were sealed by a parafilm and incubated at different temperatures 20, 25 , and $30^{\circ} \mathrm{C}$. Each treatment had 3 replicates. Control treatment only contained water. Mortality rate was assessed after 24, 48, and $72 \mathrm{~h}$ of exposure.

Table 3 Influence of different bacterial fractions on the mortality of termites in relation to temperature and time duration by spray method

\begin{tabular}{|c|c|c|c|c|c|c|c|c|c|c|}
\hline \multirow[t]{3}{*}{ Treatment } & \multirow[t]{3}{*}{ Conc. } & \multicolumn{9}{|c|}{ Mortality\% \pm SD } \\
\hline & & \multicolumn{3}{|l|}{$20^{\circ} \mathrm{C}$} & \multicolumn{3}{|l|}{$25^{\circ} \mathrm{C}$} & \multicolumn{3}{|l|}{$30^{\circ} \mathrm{C}$} \\
\hline & & $24 \mathrm{~h}$ & $48 \mathrm{~h}$ & $72 \mathrm{~h}$ & $24 \mathrm{~h}$ & $48 \mathrm{~h}$ & $72 \mathrm{~h}$ & $24 \mathrm{~h}$ & $48 \mathrm{~h}$ & $72 \mathrm{~h}$ \\
\hline \multirow[t]{3}{*}{$\begin{array}{l}T_{1}, \text { Xenorhabdus indica } \\
\text { (Pak.S.B.50) }\end{array}$} & B.S. & $\begin{array}{l}83.66 \pm \\
7.44 a\end{array}$ & $\begin{array}{l}100 \pm \\
0.00 c\end{array}$ & - & $\begin{array}{l}80.66 \pm \\
6.98 a\end{array}$ & $\begin{array}{l}100 \pm \\
0.00 c\end{array}$ & - & $\begin{array}{l}71.66 \pm \\
6.44 a\end{array}$ & $\begin{array}{l}78.66 \pm \\
6.75 a\end{array}$ & $\begin{array}{l}78.66 \pm \\
7.83 a\end{array}$ \\
\hline & CFF & $\begin{array}{l}88.33 \pm \\
9.34 a\end{array}$ & $\begin{array}{l}100 \pm \\
0.00 c\end{array}$ & - & $\begin{array}{l}87.33 \pm \\
7.45 a\end{array}$ & $\begin{array}{l}100 \pm \\
0.00 c\end{array}$ & - & $\begin{array}{l}77.33 \pm \\
8.54 a\end{array}$ & $\begin{array}{l}82.33 \pm \\
7.35 a\end{array}$ & $\begin{array}{l}82.33 \pm \\
8.41 \mathrm{a}\end{array}$ \\
\hline & B.R. & $\begin{array}{l}34.66 \pm \\
5.34 b\end{array}$ & $\begin{array}{l}45.66 \pm \\
5.41 b\end{array}$ & $\begin{array}{l}45.33 \pm \\
7.23 b\end{array}$ & $\begin{array}{l}32.33 \pm \\
7.33 b\end{array}$ & $\begin{array}{l}45.66 \pm \\
6.76 b\end{array}$ & $\begin{array}{l}47.33 \pm \\
9.21 b\end{array}$ & $\begin{array}{l}24.66 \pm \\
5.20 \mathrm{~b}\end{array}$ & $\begin{array}{l}29.66 \pm \\
5.67 b\end{array}$ & $\begin{array}{l}33.33 \pm \\
5.75 b\end{array}$ \\
\hline \multirow[t]{3}{*}{$\begin{array}{l}T_{2}, \text { Xenorhabdus indica } \\
\text { (Pak.S.B.56) }\end{array}$} & B.S. & $\begin{array}{l}85.66 \pm \\
7.34 a\end{array}$ & $\begin{array}{l}100 \pm \\
0.00 c\end{array}$ & - & $\begin{array}{l}80.33 \pm \\
8.34 a\end{array}$ & $\begin{array}{l}83.33 \pm \\
9.38 a\end{array}$ & $\begin{array}{l}92.33 \pm \\
6.39 c\end{array}$ & $\begin{array}{l}74.66 \pm \\
6.78 a\end{array}$ & $\begin{array}{l}77.33 \pm \\
6.78 a\end{array}$ & $\begin{array}{l}81.33 \pm \\
6.39 a\end{array}$ \\
\hline & CFF & $\begin{array}{l}98.33 \pm \\
6.22 \mathrm{c}\end{array}$ & $\begin{array}{l}100 \pm \\
0.00 c\end{array}$ & - & $\begin{array}{l}87.33 \pm \\
8.32 a\end{array}$ & $\begin{array}{l}88.66 \pm \\
7.57 a\end{array}$ & $\begin{array}{l}95.33 \pm \\
9.34 c\end{array}$ & $\begin{array}{l}78.33 \pm \\
7.43 a\end{array}$ & $\begin{array}{l}80.33 \pm \\
7.56 a\end{array}$ & $\begin{array}{l}82.33 \pm \\
8.45 a\end{array}$ \\
\hline & B.R. & $\begin{array}{l}35.66 \pm \\
4.56 b\end{array}$ & $\begin{array}{l}37.33 \pm \\
5.22 b\end{array}$ & $\begin{array}{l}37.33 \pm \\
5.22 \mathrm{~b}\end{array}$ & $\begin{array}{l}34.66 \pm \\
8.55 b\end{array}$ & $\begin{array}{l}35.33 \pm \\
6.56 b\end{array}$ & $\begin{array}{l}39.33 \pm \\
6.98 b\end{array}$ & $\begin{array}{l}28.66 \pm \\
4.56 b\end{array}$ & $\begin{array}{l}32.33 \pm \\
4.87 \mathrm{~b}\end{array}$ & $\begin{array}{l}35.33 \pm \\
5.43 \mathrm{~b}\end{array}$ \\
\hline \multirow[t]{3}{*}{$\begin{array}{l}T_{3}, \text { Xenorhabdus } \\
\text { stockiae(Pak.S.B. 65) }\end{array}$} & B.S. & $\begin{array}{l}85.66 \pm \\
8.23 a\end{array}$ & $\begin{array}{l}100 \pm \\
0.00 c\end{array}$ & - & $\begin{array}{l}78.66 \pm \\
8.23 a\end{array}$ & $\begin{array}{l}73.66 \pm \\
9.26 a\end{array}$ & $\begin{array}{l}78.97 \pm \\
7.87 a\end{array}$ & $\begin{array}{l}69.66 \pm \\
6.87 a\end{array}$ & $\begin{array}{l}72.66 \pm \\
9.45 a\end{array}$ & $\begin{array}{l}79.66 \pm \\
8.09 a\end{array}$ \\
\hline & CFF & $\begin{array}{l}88.33 \pm \\
7.22 \mathrm{a}\end{array}$ & $\begin{array}{l}100 \pm \\
0.00 c\end{array}$ & - & $\begin{array}{l}88.33 \pm \\
7.22 \mathrm{a}\end{array}$ & $\begin{array}{l}100 \pm \\
0.00 c\end{array}$ & - & $\begin{array}{l}72.33 \pm \\
8.49 a\end{array}$ & $\begin{array}{l}75.33 \pm \\
7.54 a\end{array}$ & $\begin{array}{l}82.33 \pm \\
9.34 a\end{array}$ \\
\hline & B.R. & $\begin{array}{l}33.66 \pm \\
4.22 b\end{array}$ & $\begin{array}{l}35.33 \pm \\
6.22 \mathrm{~b}\end{array}$ & $\begin{array}{l}38.43 \pm \\
4.10 \mathrm{~b}\end{array}$ & $\begin{array}{l}29.33 \pm \\
5.35 b\end{array}$ & $\begin{array}{l}30.66 \pm \\
5.67 b\end{array}$ & $\begin{array}{l}38.66 \pm \\
4.56 b\end{array}$ & $\begin{array}{l}25.66 \pm \\
3.86 b\end{array}$ & $\begin{array}{l}35.33 \pm \\
6.22 \mathrm{~b}\end{array}$ & $\begin{array}{l}38.43 \pm \\
4.10 \mathrm{~b}\end{array}$ \\
\hline \multirow[t]{3}{*}{$\begin{array}{l}T_{4}, \text { Xenorhabdus } \\
\text { steinernematis (C.B.10) }\end{array}$} & B.S. & $\begin{array}{l}100 \pm \\
0.00 c\end{array}$ & - & - & $\begin{array}{l}100 \pm \\
0.00 c\end{array}$ & - & - & $\begin{array}{l}87.66 \pm \\
9.45 a\end{array}$ & $\begin{array}{l}100 \pm \\
0.00 c\end{array}$ & - \\
\hline & CFF & $\begin{array}{l}100 \pm \\
0.00 c\end{array}$ & - & - & $\begin{array}{l}100 \pm \\
0.00 c\end{array}$ & - & - & $\begin{array}{l}93.33 \pm \\
8.22 \mathrm{C}\end{array}$ & $\begin{array}{l}100 \pm \\
0.00 c\end{array}$ & - \\
\hline & B.R. & $\begin{array}{l}36.33 \pm \\
3.43 b\end{array}$ & $\begin{array}{l}39.33 \pm \\
6.37 b\end{array}$ & $\begin{array}{l}42.33 \pm \\
6.36 \mathrm{~b}\end{array}$ & $\begin{array}{l}34.33 \pm \\
3.03 b\end{array}$ & $\begin{array}{l}35.66 \pm \\
4.67 b\end{array}$ & $\begin{array}{l}37.33 \pm \\
3.65 b\end{array}$ & $\begin{array}{l}26.33 \pm \\
3.65 b\end{array}$ & $\begin{array}{l}27.33 \pm \\
4.55 b\end{array}$ & $\begin{array}{l}28.33 \pm \\
5.43 b\end{array}$ \\
\hline Control & $\mathrm{H}_{2} \mathrm{O}$ & $\begin{array}{l}0.00 \pm \\
.00 \mathrm{~d}\end{array}$ & $\begin{array}{l}0.00 \pm \\
.00 d\end{array}$ & $\begin{array}{l}0.00 \pm \\
.00 \mathrm{~d}\end{array}$ & $\begin{array}{l}0.00 \pm \\
.00 d\end{array}$ & $\begin{array}{l}0.00 \pm \\
.00 \mathrm{~d}\end{array}$ & $\begin{array}{l}0.00 \pm \\
.00 \mathrm{~d}\end{array}$ & $\begin{array}{l}0.00 \pm \\
.00 \mathrm{~d}\end{array}$ & $\begin{array}{l}0.00 \pm \\
.00 \mathrm{~d}\end{array}$ & $\begin{array}{l}0.00 \pm \\
.00 d\end{array}$ \\
\hline
\end{tabular}

B.S. bacterial suspension $\left(4 \times 10^{4} \mathrm{CFU} / \mathrm{ml}\right)$, CFF cell-free filtrate $(100 \mu \mathrm{l} / 10 \mathrm{ml})$, B.R. bacterial residue $(100 \mu \mathrm{l} / 10 \mathrm{ml})$, "-" experiment complete The similar alphabets in rows and columns have shown non significant differences at $p=0.01$ 


\section{Data analysis}

Data are expressed as means, standard deviation and the significance of mean differences was determined with Duncan's multiple range test (SAS Institute, Cary, NC).

\section{Results and discussion}

\section{Biochemical analysis}

The biochemical test of Xenorhabdus species were assessed for the following features. Citrate utilization, esculin hydrolysis, catalase, meso-inositol fermentation, salicin fermentation, ribose fermentation, and lipase tween 80. X. indica Pak.S.B.50 showed 90$100 \%$ positive expression in all analysis, except for Esculin hydrolysis. X. indica Pak.S.B.56 contained Citrate utilization; Esculin hydrolysis and Lipase Tween 80 resulted in $90-100 \%$ positive and catalase $75-89 \%$ positive, whereas meso-inositol fermentation, Salicin fermentation, and ribose fermentation $90-100 \%$ negative. X. stockiae Pak.S.B. 65 had $25-74 \%$ positive results for meso-inositol fermentation where all remaining factors were found to be $90-100 \%$ negative.
X. steinernematis C.B.10 expressed $90-100 \%$ positive for all examined biochemical tests but negative 90$100 \%$ for Salicin fermentation (Table 2).

\section{Effect of different application methods for controlling Microtermes species Spray method}

All bacterial isolates were found to be significantly effective against termites by spray method. Different fractions of bacterial formulations showed significant differences of mortality rate $(P<0.001)$. Bacterial suspension and cell-free filtrates of all treatments (bacterial isolates) had the potential to control termites at 20 and $25^{\circ} \mathrm{C}$ even after $24 \mathrm{~h}$, whereas the bacterial residue of all the bacterial isolates had least potential for controlling termites. Due to direct contact of formulations with termites, effective results were obtained within $24 \mathrm{~h}$ in most of the cases. Mortality response of cell-free filtrates after $24 \mathrm{~h}$ at $20^{\circ} \mathrm{C}$ in Xenorhabdus indica (Pak.S.B.50), $T_{2}(X$. indica (Pak.S.B.56)), $T_{3}$ (X. stockiae (Pak.S.B. 65)), and

Table 4 Influence of different bacterial fractions on the mortality of termites in relation to temperature and time duration by sand barrier assay method

\begin{tabular}{|c|c|c|c|c|c|c|c|c|c|c|}
\hline \multirow[t]{3}{*}{ Treatment } & \multirow[t]{3}{*}{ Conc. } & \multicolumn{9}{|c|}{ Mortality \% } \\
\hline & & \multicolumn{3}{|l|}{$20^{\circ} \mathrm{C}$} & \multicolumn{3}{|l|}{$25^{\circ} \mathrm{C}$} & \multicolumn{3}{|l|}{$30^{\circ} \mathrm{C}$} \\
\hline & & $24 \mathrm{~h}$ & $48 \mathrm{~h}$ & $72 \mathrm{~h}$ & $24 \mathrm{~h}$ & $48 \mathrm{~h}$ & $72 \mathrm{~h}$ & $24 \mathrm{~h}$ & $48 \mathrm{~h}$ & $72 \mathrm{~h}$ \\
\hline \multirow[t]{3}{*}{$\begin{array}{l}T_{1}, \text { Xenorhabdus indica } \\
\text { (Pak.S.B.50) }\end{array}$} & B.S. & $\begin{array}{l}82.66 \pm \\
6.98 a\end{array}$ & $\begin{array}{l}100 \pm \\
0.00 c\end{array}$ & - & $\begin{array}{l}85.66 \pm \\
8.56 a\end{array}$ & $\begin{array}{l}100 \pm \\
0.00 c\end{array}$ & - & $\begin{array}{l}73.33 \pm \\
5.98 a\end{array}$ & $\begin{array}{l}76.66 \pm \\
7.87 a\end{array}$ & $\begin{array}{l}80.66 \pm \\
6.88 a\end{array}$ \\
\hline & CFF & $\begin{array}{l}87.33 \pm \\
7.98 \mathrm{a}\end{array}$ & $\begin{array}{l}100 \pm \\
0.00 c\end{array}$ & - & $\begin{array}{l}87.33 \pm \\
7.45 a\end{array}$ & $\begin{array}{l}100 \pm \\
0.00 c\end{array}$ & - & $\begin{array}{l}78.33 \pm \\
9.12 \mathrm{a}\end{array}$ & $\begin{array}{l}84.33 \pm \\
7.35 a\end{array}$ & $\begin{array}{l}85.33 \pm \\
8.41 a\end{array}$ \\
\hline & B.R. & $\begin{array}{l}33.66 \pm \\
6.45 b\end{array}$ & $\begin{array}{l}45.66 \pm \\
5.41 b\end{array}$ & $\begin{array}{l}47.33 \pm \\
6.87 b\end{array}$ & $\begin{array}{l}32.33 \pm \\
7.33 b\end{array}$ & $\begin{array}{l}45.66 \pm \\
6.76 b\end{array}$ & $\begin{array}{l}47.33 \pm \\
9.21 b\end{array}$ & $\begin{array}{l}23.66 \pm \\
5.20 b\end{array}$ & $\begin{array}{l}30.66 \pm \\
5.67 b\end{array}$ & $\begin{array}{l}35.33 \pm \\
5.75 b\end{array}$ \\
\hline \multirow[t]{3}{*}{$\begin{array}{l}T_{2}, \text { Xenorhabdus indica } \\
\text { (Pak.S.B.56) }\end{array}$} & B.S. & $\begin{array}{l}88.66 \pm \\
7.34 \mathrm{a}\end{array}$ & $\begin{array}{l}100 \pm \\
0.00 c\end{array}$ & - & $\begin{array}{l}80.33 \pm \\
8.34 a\end{array}$ & $\begin{array}{l}83.33 \pm \\
9.38 \mathrm{a}\end{array}$ & $\begin{array}{l}92.33 \pm \\
6.39 c\end{array}$ & $\begin{array}{l}72.66 \pm \\
6.78 a\end{array}$ & $\begin{array}{l}78.33 \pm \\
6.78 a\end{array}$ & $\begin{array}{l}85.33 \pm \\
6.39 a\end{array}$ \\
\hline & CFF & $\begin{array}{l}93.33 \pm \\
6.22 \mathrm{C}\end{array}$ & $\begin{array}{l}100 \pm \\
0.00 c\end{array}$ & - & $\begin{array}{l}88.33 \pm \\
7.76 a\end{array}$ & $\begin{array}{l}88.66 \pm \\
7.57 a\end{array}$ & $\begin{array}{l}95.33 \pm \\
9.34 c\end{array}$ & $\begin{array}{l}75.33 \pm \\
7.43 a\end{array}$ & $\begin{array}{l}82.33 \pm \\
7.56 a\end{array}$ & $\begin{array}{l}85.33 \pm \\
8.45 a\end{array}$ \\
\hline & B.R. & $\begin{array}{l}37.66 \pm \\
4.56 b\end{array}$ & $\begin{array}{l}38.33 \pm \\
5.22 b\end{array}$ & $\begin{array}{l}39.33 \pm \\
5.22 \mathrm{~b}\end{array}$ & $\begin{array}{l}34.33 \pm \\
5.34 b\end{array}$ & $\begin{array}{l}35.33 \pm \\
6.56 b\end{array}$ & $\begin{array}{l}39.33 \pm \\
6.98 b\end{array}$ & $\begin{array}{l}26.66 \pm \\
4.56 b\end{array}$ & $\begin{array}{l}36.33 \pm \\
4.87 b\end{array}$ & $\begin{array}{l}39.33 \pm \\
5.43 b\end{array}$ \\
\hline \multirow[t]{3}{*}{$\begin{array}{l}T_{3}, \text { Xenorhabdus } \\
\text { stockiae(Pak.S.B.65) }\end{array}$} & B.S. & $\begin{array}{l}88.66 \pm \\
7.98 a\end{array}$ & $\begin{array}{l}100 \pm \\
0.00 c\end{array}$ & - & $\begin{array}{l}78.66 \pm \\
8.23 a\end{array}$ & $\begin{array}{l}73.66 \pm \\
9.26 a\end{array}$ & $\begin{array}{l}78.97 \pm \\
7.87 a\end{array}$ & $\begin{array}{l}65.66 \pm \\
6.87 a\end{array}$ & $\begin{array}{l}75.66 \pm \\
9.45 a\end{array}$ & $\begin{array}{l}75.66 \pm \\
8.09 a\end{array}$ \\
\hline & CFF & $\begin{array}{l}85.33 \pm \\
8.65 a\end{array}$ & $\begin{array}{l}100 \pm \\
0.00 c\end{array}$ & - & $\begin{array}{l}86.33 \pm \\
7.22 \mathrm{a}\end{array}$ & $\begin{array}{l}100 \pm \\
0.00 c\end{array}$ & - & $\begin{array}{l}71.33 \pm \\
8.49 a\end{array}$ & $\begin{array}{l}75.33 \pm \\
7.54 \mathrm{a}\end{array}$ & $\begin{array}{l}84.33 \pm \\
9.34 a\end{array}$ \\
\hline & B.R. & $\begin{array}{l}34.66 \pm \\
4.22 b\end{array}$ & $\begin{array}{l}35.33 \pm \\
6.22 b\end{array}$ & $\begin{array}{l}38.43 \pm \\
4.10 b\end{array}$ & $\begin{array}{l}30.33 \pm \\
5.35 b\end{array}$ & $\begin{array}{l}34.66 \pm \\
5.67 b\end{array}$ & $\begin{array}{l}38.66 \pm \\
4.56 b\end{array}$ & $\begin{array}{l}28.66 \pm \\
3.86 b\end{array}$ & $\begin{array}{l}35.33 \pm \\
6.22 b\end{array}$ & $\begin{array}{l}40.43 \pm \\
4.10 b\end{array}$ \\
\hline \multirow[t]{3}{*}{$\begin{array}{l}T_{4}, \text { Xenorhabdus } \\
\text { steinernematis (C.B.10) }\end{array}$} & B.S. & $\begin{array}{l}100 \pm \\
0.00 c\end{array}$ & - & - & $\begin{array}{l}100 \pm \\
0.00 c\end{array}$ & - & - & $\begin{array}{l}83.66 \pm \\
9.45 a\end{array}$ & $\begin{array}{l}100 \pm \\
0.00 c\end{array}$ & - \\
\hline & CFF & $\begin{array}{l}100 \pm \\
0.00 c\end{array}$ & - & - & $\begin{array}{l}100 \pm \\
0.00 c\end{array}$ & - & - & $\begin{array}{l}96.33 \pm \\
8.22 c\end{array}$ & $\begin{array}{l}100 \pm \\
0.00 c\end{array}$ & - \\
\hline & B.R. & $\begin{array}{l}38.33 \pm \\
3.43 b\end{array}$ & $\begin{array}{l}40.33 \pm \\
6.37 b\end{array}$ & $\begin{array}{l}44.33 \pm \\
7.32 \mathrm{~b}\end{array}$ & $\begin{array}{l}35.33 \pm \\
3.03 b\end{array}$ & $\begin{array}{l}36.66 \pm \\
4.67 b\end{array}$ & $\begin{array}{l}37.66 \pm \\
4.87 b\end{array}$ & $\begin{array}{l}26.33 \pm \\
3.65 b\end{array}$ & $\begin{array}{l}28.33 \pm \\
4.55 b\end{array}$ & $\begin{array}{l}34.33 \pm \\
5.43 b\end{array}$ \\
\hline Control & $\mathrm{H}_{2} \mathrm{O}$ & $\begin{array}{l}0.00 \pm \\
.00 d\end{array}$ & $\begin{array}{l}0.00 \pm \\
.00 d\end{array}$ & $\begin{array}{l}0.00 \pm \\
.00 \mathrm{~d}\end{array}$ & $\begin{array}{l}0.00 \pm \\
.00 \mathrm{~d}\end{array}$ & $\begin{array}{l}0.00 \pm \\
.00 d\end{array}$ & $\begin{array}{l}0.00 \pm \\
.00 \mathrm{~d}\end{array}$ & $\begin{array}{l}0.00 \pm \\
.00 d\end{array}$ & $\begin{array}{l}0.00 \pm \\
.00 d\end{array}$ & $\begin{array}{l}0.00 \pm \\
.00 d\end{array}$ \\
\hline
\end{tabular}

B.S. bacterial suspension $\left(4 \times 10^{4} \mathrm{CFU} / \mathrm{ml}\right)$, CFF cell-free filtrate $(100 \mu \mathrm{l} / 10 \mathrm{ml})$, B.R. bacterial residue $(100 \mu \mathrm{l} / 10 \mathrm{ml})$, "_" experiment complete 
$T_{4}$ (X. steinernematis (C.B.10)) ranged between 88.3 and $100 \%$ as $88.33 \pm 9.34,98.33 \pm 6.22,88.33 \pm 7.22$, and $100.00 \% \pm 0.00$, respectively. No mortality response was found in the control treatment (Table 3).

\section{Sand barrier assay}

In a sand barrier assay, Pakistani isolates of EPB were applied $20 \mathrm{ml}$ of different formulations: B.S. (bacterial suspension) $\left(4 \times 10^{4} \mathrm{CFU} / \mathrm{ml}\right), \mathrm{CFF}$ (cell free filtrate) $(100 \mu \mathrm{l} / 10 \mathrm{ml})$, B.R. (bacterial residue) $(100 \mu \mathrm{l} / 10 \mathrm{ml})$ of $T_{1}$ (Xenorhabdus indica (Pak.S.B.50)), $T_{2}$ (X. indica (Pak.S.B.56)), $T_{3}$ (X. stockiae (Pak.S.B. 65)), and $T_{4}(X$. steinernematis (C.B.10)). Significant differences were observed between control and treatments $(P<0.001)$. Effectiveness of different formulations was dependent on the temperature and time duration. The most effective treatment was $T_{4}$, where maximum percentage of mortality found to be $100 \%$ after $24 \mathrm{~h}$ of application by B.S. $\left(4 \times 10^{4} \mathrm{CFU} / \mathrm{ml}\right)$; CFF $(100 \mu \mathrm{l} / 10 \mathrm{ml})$ at 20 and $25^{\circ}$ C. After applying $T_{1}$ (Xenorhabdus indica (Pak.S.B.50)), $T_{2}$ (X. indica (Pak.S.B.56)), and $T_{3}$ (X. stockiae (Pak.S.B. 65)) 100\% mortality of termites was obtained after $48 \mathrm{~h}$ in B.S. $\left(4 \times 10^{4} \mathrm{CFU} / \mathrm{ml}\right)$ and CFF $(100 \mu \mathrm{l} / 10 \mathrm{ml})$, whereas all insects survived in control treatment (Table 4).

Two different application methods were determined for their proficiency and significantly similar results obtained from both methods.

Different bacterial species have the capability to control termites. The effects of Bacillus thuringiensis subspecies was examined under laboratory conditions against Nasutitermes ehrhardti (Castilhos-Fortes et al. 2002). They observed that $B$. thuringiensis subspecies kurstaki produced $80 \%$ mortality of termite species. $B$. thuringiensis proteins having insecticidal properties are highly specific as gut toxins and it has shown a superior safety in reference to the effectiveness for non-target organism (Sarwar, 2015).

Pseudomonas sp., P.maltophilia, Bacillus strains, and Paenibacillus sp., are reported to produce chitinase (Suyal et al., 2015; Verma et al., 2016a; Yadav et al., 2016a). It was also reported that ten bacterial strains along with two control strains have been evaluated as biocontrol against termites. Different bacterial strains having termite-killing ability showed $>80 \%$ mortality after 5 days of incubation (Dua, 2014). Four bacterial strains caused $100 \%$ killing at 10 days of observation. The cell-free culture filtrate of these cultures showed that the antagonistic substance was extracellular having protein properties. Bacterial strains of Bacillus subtilis KBM79 and Pseudomonas synxantha KPM35 possessed proteolytic, chitinolytic, and lipolytic enzyme activities and caused 100\% killing of termites (Yadav et al., 2016b). In previous studies, $X$. nematophila has been proved as a potential candidate of biocontrol agent against termites (Hiranwrongwera et al., 2007).

\section{Conclusion}

The present results proved that different bacterial fractions of Xenorhabdus species were found effective against termites in certain adequate conditions and can be a successful candidate for integration in termites' controlling strategy.

\section{Abbreviations}

B.S.: Bacterial suspension; CFF: Cell-free filtrate; B.R.: Bacterial residue

\section{Acknowledgements}

Special thanks to Dr. Shumaiza, Dept. of Genetics, University of Karachi, Karachi, Pakistan, for providing lab facilities to conduct the biochemical test.

\section{Authors' contributions}

EYI planned the research experiment and performed biochemical analysis and mortality response. UAM and SFZA managed data and analyzed and interpreted the results. SR helped in writing the manuscript and reviewed the manuscript. SF provided the EPN material and supervised this research. All authors have read and approved the final manuscript.

\section{Funding}

Our gratitude goes to the Pakistan Science Foundation (PSF) for providing the funds to conduct the project (084) under which this research has been conducted.

\section{Availability of data and materials}

The datasets used and/or analyzed during the current study are available from the corresponding author on reasonable request.

Ethics approval and consent to participate

N/A

\section{Consent for publication}

N/A

\section{Competing interests}

The authors declare that they have no competing interests.

Received: 31 January 2020 Accepted: 5 March 2020

Published online: 17 March 2020

\section{References}

Akhurst R (1980) Morphological and functional dimorphism in Xenorhabdus spp., bacteria symbiotically associated with the insect pathogenic nematodes Neoaplectana and Heterorhabditis. J. Gen. Microbiol 121:303-309. https://doi. org/10.1099/00221287-121-2-303

Bignell DE, Eggleton P (1998) Termites. In: Calow P (ed) Encyclopedia of ecology and environmental management. Blackwell Scientific, Oxford, pp 744-746

Castilhos-Fortes R, Matsumura ATS, Diehl E, Fiuza LM (2002) Susceptibility of Nasutitermes ehrhardti (Isoptera: Termitidae) to Bacillus thuringiensis subspecies. Brazi J Microbiol 33:219-222

Dua S (2014). Biological control of subterranean termites (Isoptera: Termitidae) with soil bacte-ria. Ph.D. thesis, Chaudhary Charan Singh Haryana Agricultural University, pp. 225.

Dutky SR, Thompson JV, Cantwell GE (1974) A technique for the mass propagation of the DD-136 nematode. J. Insect Pathol 6:417-422

Fujii JK (1975) Effect of an entomogenous nematode Neoaplectana carpocapsae Weiser, on the Formosan subterranean termite, Captotermes formosanus Shiraki, with ecological and biological studies on C. formosanus. University of Hawaii, Honolulu, Hawaii, USA, Ph.D. dissertation, p 163

Georgis R, Poinar GO Jr, Wilson AP (1982) Susceptibility of damp-wood termites and soil and wood-dwelling termites to the entomogenous nematode Neoaplectana carpocapsae. IRCS Medical Science 10:563 
Govorushko SM (2011) Biodeterioration: biological processes. In: Natura processes and human impacts: interactions between humanity and the environment. Springer, p 335

Hiranwrongwera C, Adisettakul P, Tansirichaiya S, Piyabun O, Somsuk V(2007). Efficiency of a nematode (Steinernema carpocapsae) and its symbiotic bacterium (Xenorhabdus nematophila) at eliminating the termite Coptotermes curvignathus that infests para rubber (Hevea brasiliensis). The 5th international symposium on biocontrol and biotechnology (p. 90). Nong Khai Campus, Nong Khai: Khon Kaen University.

Laumond C, Mauleon H, Kermarrec A (1979) Donn_ees nouvelles sur le spectre d'ho^tes et le parasitisme du n_ematode entomophage Neoaplectana carpocapsae. Entomophaga 24:13-27

Manzoor F, Naeem M (2010). Survey of termite infested houses, indigenous building materials and construction techniques in Pakistan. Pak J Zoology42, 6: 693-696.

Noirot C, Noirot-Timothee C (1970) The digestive system. In: Weesner FM (ed) Biology of termites Krishna, K. Academic, New York and London, pp 49-88

Sarwar, M (2015). Microbial insecticides-an ecofriendly effective line of attack for insect pests management. Inter J Engi Adv Res Technol1, 4-9.

Suyal DC, Yadav A, Shouche Y, Goel R (2015) Bacterial diversity and community structure of Western Indian Himalayan red kidney bean (Phaseolus vulgaris) rhizosphere as revealed by 165 rRNA gene sequences. Biologia 70:305-313

Trudeau D (1989) Selection of entomophilic nematodes for control of the eastern subterranean termite, Reticultermes flavipes (Kollar) (Isoptera: Rhinotermitidae) University of Toronto, Toronto, Ontario, Canada, Master's thesis, p 93

Verma M, Sharma S, Prasad R (2009) Biological alternatives for termite control: a review. Int. Biodeter Biodegradation 63:959-972

Verma P, Yadav AN, Khannam KS, Kumar S, Saxena AK, Suman A (2016a). Molecular diversity and multifarious plant growth promoting attributes of Bacilli associated with wheat (Triticum aestivum L.) rhizosphere from six diverse agro-ecological zones of India. Journal of Basic Microbiology56, 4458.

White GF (1927) A method for obtaining infective nematode larvae from cultures. Science 66:302-303

Yadav AN, Sachan SG, Verma P, Kaushik R, Saxena AK (2016a) Cold active hydrolytic enzymes production by psychrotrophic Bacilli isolated from three subglacial lakes of NW Indian Himalayas. Journal of Basic Microbiology 56:294307

Yadav AN, Sachan SG, Verma P, Saxena AK (2016b) Bioprospecting of plant growth promoting psychrotrophic Bacilli from cold desert of north western Indian Himalayas. Indian Journal of Experimental Biology 54:142-150

\section{Publisher's Note}

Springer Nature remains neutral with regard to jurisdictional claims in published maps and institutional affiliations.

\section{Submit your manuscript to a SpringerOpen ${ }^{\circ}$ journal and benefit from:}

- Convenient online submission

- Rigorous peer review

- Open access: articles freely available online

- High visibility within the field

- Retaining the copyright to your article

Submit your next manuscript at $\boldsymbol{\nabla}$ springeropen.com 